\title{
Anti-convulsant Hypersensitivity Syndrome with Predominant Liver Involvement
}

\author{
Gul Javid, D.M., Syed Mushtaq Saif, M.D., Abid Shoukat, M.D., Riyaz u Saif, M.D., Mohsin ul \\ Rasool, M.D., Khursheed Ahmad, M.D., Yasin Mujoo, M.D., Shaheen Parveen, M.D., Asif lqball, D.M \\ Department of Gastroenterology, Sher-i-Kashmir Institute of Medical Sciences, Post Bag 27, Soura, Srinagar- 190011 , J\&K India.
}

\section{A B S T R A C T}

Anticonvulsant hypersensitivity syndrome (AHS) is a rare but potentially life threatening syndrome that develops in genetically susceptible individuals following exposure to aromatic antiepileptic drugs (AED's) like phenytoin. It is characterized by fever, rash and internal organ involvement. AHS should be recognized at the earliest because continuation of the culprit drug can lead to fatal outcome. We present severe phenytoin hypersensitivity syndrome with predominant hepatic involvement that improved with drug withdrawal and other supportive treatment. JMS 2011 ; 14(1):22-24

Key Words: Anticonvulsant hypersensitivity syndrome, phenytoin, adverse drug reaction, hepatitis

Anticonvulsant hypersensitivity syndrome, an immunologic reaction associated with the aromatic antiepileptic drugs, is a rare but potentially life threatening syndrome. It is characterized by the classical triad of fever, rash and internal organ involvement that develops 2-6 weeks after the exposure to the drugs. The diagnosis is a clinical one and management is withdrawal of the culprit drug. Role of steroids, intravenous immunoglobulin, N-Acetylcysteine and other agents is not yet clearly established. We present severe phenytoin hypersensitivity syndrome with hepatitis that improved with drug withdrawal, intravenous antihistamines and steroids.

Correspondence and reprint requests to:

Dr. Gul Javid

Professor and Head, Department of Gastroenterology

Sher-i-Kashmir Institute of Medical Sciences, Post Bag 27, Soura, Srinagar - 190011 , J\&K India.

Phone: 0194-2401013-16 (Ext. 2270)

guljavid@rediffmail.com

\section{Case 1}

A 13 year old male patient was admitted to our department with rash, fever and jaundice of one week duration. Fifteen days prior to this admission, the patient has been started on oral phenytoin therapy $(200 \mathrm{mg} /$ day) for newly detected generalized tonic clonic convulsions. There was no history of atopy. On examination the patient was conscious oriented, ill looking with axillary temperature of $101^{\circ} \mathrm{F}$, pulse 120 beats/min and blood pressure of 100/78mmhg. He had icterus and generalized erythematous maculopapular rash(Fig. 1). Buccal mucosa was also erythematous; however, there were no ulcers. He was having firm tender enlarged nodes in cervical, axillary and inguinal regions, without hepatomegaly or Splenomegaly. Initial impression was of an infection like viral hepatitis, drug induced liver injury and hematological malignancy. His heamogram showed leucocytosis with eosinophilia; Liver function test (LFT)revealed alkaline phosphatase(ALP) $612 \mathrm{UI} / \mathrm{L}$ (range 40-150), alanine aminotransferace (ALT) $576 \mathrm{UI} / \mathrm{L}$ (range 040), aspartate amininotransferase(AST) 634UI/L(range 0- 


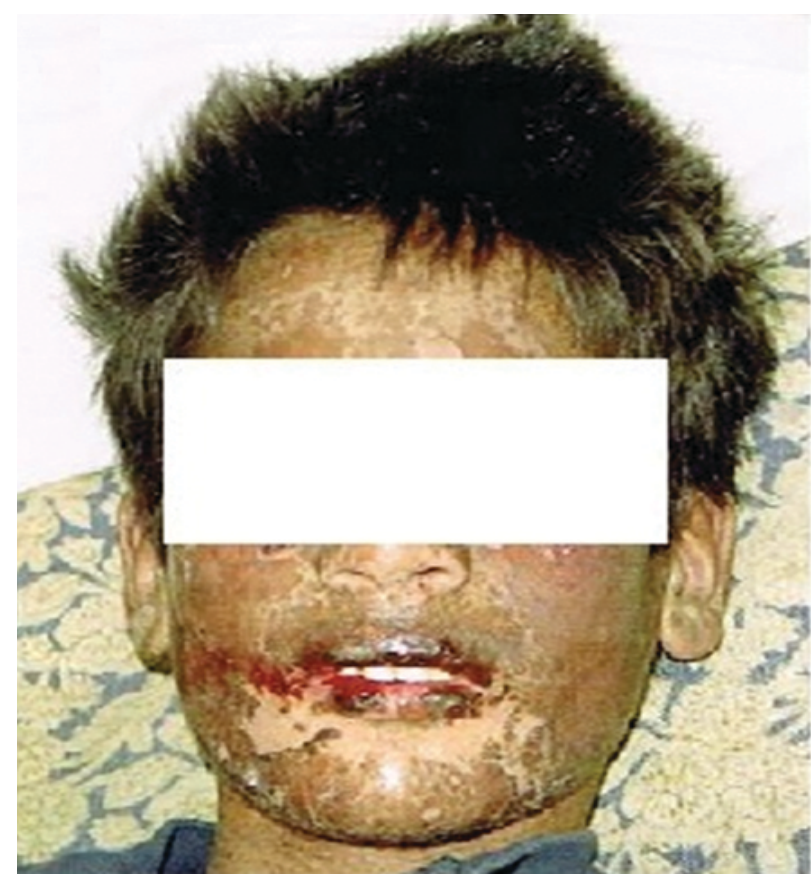

Figure 1: Before treatment showing erythematous maculopapular rash

40) and bilirubin of $9 \mathrm{mg} \%$.Kidney function test, blood gases, electrolytes and coagulogram were normal. Ultrasonography (US) showed slightly hypoechoic liver with normal biliary tree, without any internal lymphadenopathy.His viral serologies for hepatitis A, B, C, D, E, EBV(Ebstian bar virus) and $\mathrm{CMV}$ (Cytomegalovirus) were negative. His Anti nucleior antibody (ANA) was negative; cultures were sterile and lymph node biopsy showed reactive hyperplasia. The patient's Phenytoin was stopped and initially managed with Intravenous(IV) fluids, IV antihistaminic and IV corticosteroids. Following the administration of these drugs, his clinical condition improved over next 3 days. On 10th day of admission rash subsided and lymphadenopathy resolved(Fig. 2). LFT also started showing improvement after 5th day of admission. Patient was discharged after two weeks on oral methyprednisolone which was tapered over seven days. Liver functions get normalized over a period of two months. No fresh seizures on follow up.

\section{Case 2}

A 50 year old female patient was admitted to our department with fever and jaundice of one week duration, with history of rash 10 days back. One month prior to this admission, the patient had head injury with brain contusion and has been started on oral phenytoin therapy $(300 \mathrm{mg} /$ day) as prophylaxis for seizures. On examination the patient was conscious oriented, ill looking with axillary temperature of $102^{\circ} \mathrm{F}$, pulse 110 beats/min and blood pressure of $130 / 80$ $\mathrm{mmHg}$. She had icterus and scar of generalized rash which she has developed 10 days back. Buccal mucosa was

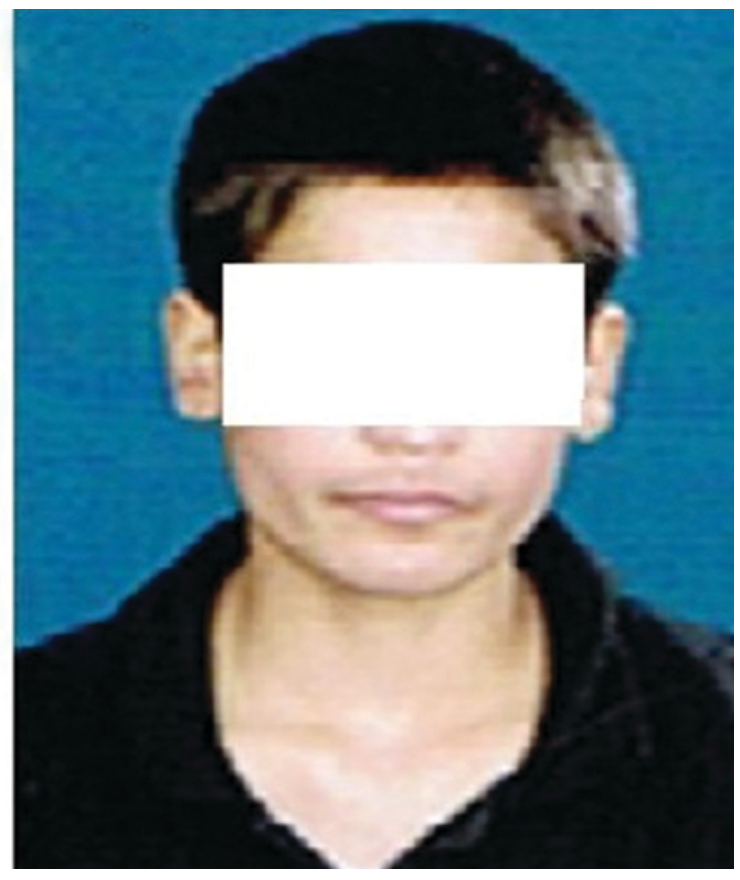

Figure 2: After treatment showing rash cleared

erythematous; however, there were no ulcers. She was having firm tender hepatomegaly. Systemic examination was unremarkable. Her heamogram showed leucocytosis with eosinophilia; LFT revealed ALP 412 UI/L, ALT 853 UI/L, AST $963 \mathrm{UI} / \mathrm{L}$ and bilirubin of $13 \mathrm{mg} \%$. Kidney function test, blood gases, electrolytes and coagulogram were normal. US showed slightly hypoechoic liver with normal biliary tree. Her viral serologies for hepatitis A, B, C, D, E, EBV and CMV were negative. Her ANA, AMA (anti-mitochondrial antibody) and ASMA (anti-smooth muscle antibody) were negative, cultures were sterile. The patient's Phenytoin was stopped and she was managed with IV fluids, IV antihistaminic. Following the administration of these drugs, her clinical condition improved over next one week, patients LFT improved and patient was discharged on multivitamins. Liver functions got normalized on follow up visits.

\section{Discussion}

In 1950, Chaikan et al described dilantin hypersensitivity syndrome as a systemic hypersensitivity reaction associated with phenytoin therapy. ${ }^{1}$ Later it was realized that other aromatic AED's like carbamazepine and phenobarbitone could also induce this syndrome and hence in 1988 it was referred as anticonvulsant hypersensitivity syndrome(AHS). ${ }^{2}$

AHS is an idiosyncratic immunologic reaction (i.e., Type-II Adverse Drug Reaction) that develops following exposure to aromatic AED's (anti-epileptic drugs) like phenytoin, carbamazepine, phenobarbitone, premonidine, zonisamide, lamotrigine and felbamate. Other drugs that sometimes induce this syndrome are sulphonamides 
(including dapsone), minocycline, terbanifine, azathioprine, allopurinol. ${ }^{3}$ The incidence of AHS is 1:1000-1:10000. ${ }^{4}$ AHS occurs mostly 2-6 weeks after the antiepileptic drug therapy is initiated. It is characterized by fever, rash, lymphadenopathy and internal organ involvement. The first signs of AHS is fever, malaise and pharyngitis (at this stage usually missed as a flu like illness). Once rash appears the diagnosis must be strongly suspected. The spectrum of liver involvement varies from mild elevation of transaminases to fulminant hepatic failure ${ }^{5}$. Severe liver involvement is an indicator of poor prognosis and mortality is as high as $18 \%$. The most common internal organ involved in AHS is liver 34-94\%. ${ }^{6}$ Blood abnormalities include leucocytosis, atypical lymphocytosis, eosinophilia, thrombocytosis, and hemolytic anemia. Less frequently other organ systems can be involved like renal (nephritis), pulmonary (Pneumonitis) has also been described. The clinical course of AHS is variable. If recognized early and the culprit drug is discontinued, the course is often less eventful, with the syndrome resolving over next few weeks.

The diagnosis of AHS is primarily clinical one (a diagnosis of exclusion) as there is no specific test available to confirm or exclude the diagnosis. AHS must be considered in any patient who has been started on aromatic AED's who present with typical triad of fever, rash and lymphadenopathy; and /or internal organ involvement. Few lab tests have been found helpful in the literature like the patch test, the leukocyte toxicity test and the leukocyte toxicity assay. ${ }^{8,9}$ However such tests have little significance for a clinician as these tests are not yet standardized and these give information in retrospection. ${ }^{10}$

The exact pathogenesis of AHS is not known yet. However it is suggested that aromatic AED's are metabolized to arene oxides, which are toxic reactive metabolites. These arene oxides are detoxified by epoxide hydroxylases. ${ }^{2}$

In the treatment of AHS, the culprit drug must be withdrawn immediately. Rest management is mainly supportive i.e., maintaining water and electrolyte balance and preventing infections. The role of corticosteroids and IVIG is controversial since controlled studies are lacking ${ }^{11}$. Intravenous IgG and plasma exchange have been successful in cases in which the disease did not regress under corticosteroids intravenous immune globulin may act by forming immune complexes that block IgG Fc receptors, by neutralizing autoantibodies, and by controlling virus infection. In some case reports, $\mathrm{N}$-acetylcysteine together withimmune globulin was successfully used. ${ }^{12}$

\section{Conclusion}

AHS should be suspected in all patients put on AED's who present with classical triad of fever, rash and internal organ involvement. The drug must be stopped immediately. Steroids may have a role but further evidence by controlled studies is needed. Selection of alternative drug is crucial and all aromatic AED's should be avoided because of high cross reactivity. Patch testing and leucocyte toxicity assay may help in identifying patients at risk of developing AHS.

\section{References}

1. Chaiken BH, Goldberg BI, Segal JP. Dilantin hypersensitivity: report of a case of hepatitis with jaundice, pyrexia, and exfoliative dermatitis. N Engl J Med 1950; 242:897-8.

2. Shear NH, Spielberg SP. Anticonvulsant hypersensitivity syndrome: in vitro assessment of risk. J Clin Invest 1988;82:1826-32.

3. Schlienger RG, Shear NH. Antiepileptic drug hypersensitivity syndrome. J Investig Allergol Clin Immunol 2006;16:321-6.

4. Tennis P, Stern RS. Risk of serious cutaneous disorders after initiation of use of Phenytoin, Carbamazepine, or Sodium Valproate; A record linkage study. Neurology 1997;49(2):542-6.

5. Chang D, Shear N. Cutaneous reactions to anticonvulsants. Semin Neurol 1992;12:329-37.

6. Powers NG, Carson NH. Idiosyncratic reactions to phenytoin. Clin Pediatr 1987;25:1204.

7. Pamela Angle, Peter Thomas, Brian Chiu and John Freedman. Bronchiolitis obliterans with organizing pneumonia and cold agglutinin disease associated with phenytoin hypersensitivity syndrome. Chest 1997;112: 1697-1699.

8. Lammintausta K, Kortekangas-Savolainen O. The usefulness of skin tests to prove drug hypersensitivity. $\mathrm{Br} J$ Dermatol 2005;152 (5):968-74.

9. Black AP. A new diagnostic method in allergic diseases. Pediatrics 1956;17(5):716-24.

10. Cohen DE, Brancaccio RR, Soter NA. Diagnostic tests for type IV or delayed hypersensitivity reactions. In; Kemp SF, Lockey RF, Editors. Diagnostic testing of allergic disease. New York: Marcel Dekker Inc., 2000: 287-305.

11. Gaedigk A, Spielberg SP, Grant DM. Characterization of the microsomal epoxide hydrolase gene in patients with anticonvulsant adverse drug reaction. Pharmacogenetics 1994; 4: 142-53.

12. Kano Y, Inaoka M, Sakuma K, Shiohara T. Virus reactivation and intravenous immunoglobulin (IVIG) therapy of drug-induced hypersensitivity syndrome. Toxicology 2005, 209:165-167. 